\title{
Strong ellipticity and spectral properties of chiral bag boundary conditions
}

\author{
C G Beneventano ${ }^{1}$, P B Gilkey ${ }^{2}$, K Kirsten ${ }^{3}$ and E M Santangelo ${ }^{1}$ \\ ${ }^{1}$ Departamento de Física, Universidad Nacional de La Plata C.C.67, 1900 La Plata, Argentina \\ ${ }^{2}$ Department of Mathematics, University of Oregon, Eugene OR 97403, USA \\ ${ }^{3}$ Department of Mathematics, Baylor University, Waco TX 76798, USA \\ E-mail: gabriela@obelix.fisica.unlp.edu.ar, gilkey@darkwing.uoregon.edu, \\ Klaus_Kirsten@Baylor.edu and mariel@obelix.fisica.unlp.edu.ar
}

Received 17 June 2003

Published 29 October 2003

Online at stacks.iop.org/JPhysA/36/11533

\begin{abstract}
We prove strong ellipticity of chiral bag boundary conditions on even dimensional manifolds. From a knowledge of the heat kernel in an infinite cylinder, some basic properties of the zeta function are analysed on cylindrical product manifolds of arbitrary even dimension.
\end{abstract}

PACS number: $02.40 . \mathrm{Vh}$

Mathematics Subject Classification: 58J50, 35P05, 35J55

\section{Introduction}

The influence that boundary conditions have on different spectral functions is an active field of research. In quantum field theory, spectral functions of particular interest are the zeta function and the heat kernel. Their dependence on the boundary condition is well understood for a large variety of boundary conditions (for a recent review see [1]). However, for some boundary conditions an understanding of elementary properties of spectral functions is still lacking. This is the case for generalized (or chiral) local bag boundary conditions [2, 3].

These boundary conditions involve an angle $\theta$, which is a substitute for introducing small quark masses to drive the breaking of chiral symmetry [3-5]. The influence the parameter $\theta$ has on various correlators was analysed in detail in [3] for the two-dimensional Euclidean ball. In [6], the heat kernel and the eta function were analysed in the two-dimensional cylinder. The situation of an arbitrary dimensional ball was considered in [7]. The results found in the above articles suggest that general properties of spectral functions, such as the pole structure of the associated zeta function and the form of the asymptotic expansion of the heat kernel, are the properties resulting from strongly elliptic local boundary conditions. For the generalized bag boundary conditions considered here, this property has not been proved. In fact, strong ellipticity is not clear at all because, for $\theta \neq 0$, the boundary conditions are 
of mixed oblique type [7] and, under certain circumstances, oblique boundary conditions are not strongly elliptic [8, 9]. However, after introducing some basic notation and properties of the boundary conditions, we will prove in sections 3 and 4 that generalized bag boundary conditions are indeed strongly elliptic boundary conditions. Based on this observation, in future investigations one might envisage a determination of heat kernel coefficients for these boundary conditions. In recent years, a conglomerate of methods has been proved to be very effective in the determination of this asymptotics [1]. Among the methods are special case considerations, which form the basis of the second half of our paper. In particular, we will determine the local heat kernel and the zeta function on cylindrical product manifolds. Results are given in terms of boundary data, much in the way it is possible for spectral boundary conditions [10]. The conclusions will summarize the main results and describe their possible future applications. We refer to [11-20] for other important related work in the field.

\section{Basic properties of chiral bag boundary conditions}

In this section, we will establish some notations for the problem at hand, i.e. the Euclidean Dirac operator acting on spinors satisfying local (chiral bag) boundary conditions [3].

Let $m=2 \bar{m}$ be even and let $P=\mathrm{i} \gamma_{j} \nabla_{j}$ be an operator of Dirac type on a compact oriented Riemannian manifold of dimension $m$. Let $V$ denote the spinor space; $\operatorname{dim}(V)=2^{\bar{m}}$. An explicit representation of the $\gamma$-matrices is provided in the appendix. They are self-adjoint and satisfy the Clifford anti-commutation relation (A.1). Near the boundary, let $e_{m}$ be the inward unit normal and $\gamma_{m}$ be the projection of the $\gamma$-matrix on the inward unit normal. In addition let $\tilde{\gamma}$ be the generalization of ' $\gamma_{5}$ ' to arbitrary even dimension, $\tilde{\gamma}=(-\mathrm{i})^{\bar{m}} \gamma_{1} \ldots \gamma_{m}$.

We set

$$
\chi=\mathrm{i} \tilde{\gamma} \mathrm{e}^{\theta \tilde{\gamma}} \gamma_{m}
$$

and use the relation $\gamma_{i} \tilde{\gamma}+\tilde{\gamma} \gamma_{i}=0$ to compute

$$
\chi^{2}=-\tilde{\gamma} \mathrm{e}^{\theta \tilde{\gamma}} \gamma_{m} \tilde{\gamma} \mathrm{e}^{\theta \tilde{\gamma}} \gamma_{m}=\tilde{\gamma} \mathrm{e}^{\theta \tilde{\gamma}} \tilde{\gamma} \mathrm{e}^{-\theta \tilde{\gamma}} \gamma_{m} \gamma_{m}=\mathbf{1}
$$

We define

$$
\Pi_{ \pm}:=\frac{1}{2}(1 \pm \chi)
$$

and have

$$
\Pi_{ \pm}^{2}=\Pi_{ \pm} \quad \text { and } \quad \Pi_{-} \Pi_{+}=\Pi_{+} \Pi_{-}=0 .
$$

Note that these two projectors are not self-adjoint (except for the particular case $\theta=0$ ). Rather, calling their respective adjoints $\Pi_{-}^{\star}$ and $\Pi_{+}^{\star}$, one has $\Pi_{ \pm}^{\star}:=\frac{1}{2}\left(1 \pm \mathrm{i} \tilde{\gamma} \mathrm{e}^{-\theta \tilde{\gamma}} \gamma_{m}\right)$, and the following equations hold:

$$
\begin{aligned}
& \Pi_{+}^{\star} \Pi_{+}=\cosh (\theta \tilde{\gamma}) \exp (-\theta \tilde{\gamma}) \Pi_{+}=\Pi_{+}^{\star} \cosh (\theta \tilde{\gamma}) \exp (-\theta \tilde{\gamma}) \\
& \Pi_{-}^{\star} \Pi_{+}=\sinh (\theta \tilde{\gamma}) \exp (-\theta \tilde{\gamma}) \Pi_{+}=\Pi_{-}^{\star} \sinh (\theta \tilde{\gamma}) \exp (-\theta \tilde{\gamma}) \\
& \Pi_{-}^{\star} \Pi_{-}=\cosh (\theta \tilde{\gamma}) \exp (-\theta \tilde{\gamma}) \Pi_{-}=\Pi_{-}^{\star} \cosh (\theta \tilde{\gamma}) \exp (-\theta \tilde{\gamma}) \\
& \Pi_{+}^{\star} \Pi_{-}=\sinh (\theta \tilde{\gamma}) \exp (-\theta \tilde{\gamma}) \Pi_{-}=\Pi_{+}^{\star} \sinh (\theta \tilde{\gamma}) \exp (-\theta \tilde{\gamma}) .
\end{aligned}
$$

We use $\Pi_{-}$to define boundary conditions for $P$. Similarly, we shall let $\mathcal{B}:=\Pi_{-} \oplus \Pi_{-} P$ define the associated boundary condition for $P^{2}$.

In the following two sections, we will show that $\left(P, \Pi_{-}\right)$and $\left(P^{2}, \mathcal{B}\right)$ define strongly elliptic boundary conditions and, as a result, we can assume standard results on the meromorphic structure of eta and zeta-invariants hold [21]. Otherwise stated, we will prove statements (1) and (2) of the following theorem. 


\section{Theorem 2.1.}

(1) $\left(P, \Pi_{-}\right)$is strongly elliptic with respect to $\mathbf{C}-\mathbf{R}^{+}-\mathbf{R}^{-}$.

(2) $\left(P^{2}, \mathcal{B}\right)$ is strongly elliptic with respect to $\mathbf{C}-\mathbf{R}^{+}$.

(3) $\left(P, \Pi_{-}\right)$is self-adjoint.

(4) $\left(P^{2}, \mathcal{B}\right)$ is self-adjoint.

Statements (3) and (4) are well known to hold [3] and so we concentrate on statements (1) and (2).

\section{Ellipticity of the first-order boundary value problem}

Proof of (1). We use lemma 1.11.2 (a) of [21] to prove assertion (1). Note that the special case $\theta=0$ defines standard mixed boundary conditions and the theorem is known to hold for this case. Let $x=\left(y_{1}, \ldots, y_{m-1}, x_{m}\right)$ be coordinates near the boundary where $x_{m}$ is the geodesic distance to the boundary and where $y=\left(y_{1}, \ldots, y_{m-1}\right)$ are coordinates on $\partial M$. Let $\xi_{a} \mathrm{~d} y^{a} \in T^{*}(\partial M)$.

Following [21] we define

$\tilde{q}(\xi, \lambda)=-\mathrm{i} \gamma_{m}\left(\sum_{a<m} \gamma_{a} \xi_{a}-\lambda\right) \quad$ for $\quad(\xi, \lambda) \neq(\overrightarrow{0}, 0) \quad$ and $\quad \lambda \notin \mathbf{R}-\{0\}$.

We then have $\tilde{q}(\xi, \lambda)^{2}=\left(|\xi|^{2}-\lambda^{2}\right) \mathbf{1}$. As $\left(|\xi|^{2}-\lambda^{2}\right) \notin \mathrm{i} \mathbf{R}$, we may let $V_{ \pm}^{\tilde{q}}$ be the span of the eigenvectors of $\tilde{q}(\xi, \lambda)$ with positive/negative real parts. We let

$$
W:=\operatorname{Kernel}\left(\Pi_{-}\right)=\operatorname{Range}\left(\Pi_{+}\right) .
$$

Using lemma 1.11.2 (a) of [21], we prove assertion (1) by verifying that $\Pi_{-}$is an isomorphism from $V_{-}^{\tilde{q}}(\xi, \lambda)$ to $\mathcal{W}=\operatorname{Range}\left(\Pi_{-}\right)$. This is equivalent to showing

$$
V_{-}^{\tilde{q}}(\xi, \lambda) \cap W=\{0\} .
$$

We change variables slightly setting $\lambda=-\mathrm{i} \mu$ where $\mu \notin \mathrm{i} \mathbf{R}-\{0\}$ and $(\xi, \mu) \neq(\overrightarrow{0}, 0)$. We then have

$$
\tilde{q}(\xi, \mu)=-\mathrm{i} \gamma_{m} \sum_{a<m} \gamma_{a} \xi_{a}+\mu \gamma_{m} \text { and } \tilde{q}(\xi, \mu)^{2}=\left(|\xi|^{2}+\mu^{2}\right) \mathbf{1}
$$

The next step in the proof is to reduce the problem to a collection of effective two-dimensional ones. We make use of the properties of the $\gamma$-matrices. First note that the elements

$$
\tau_{1}:=\mathrm{i} \gamma_{2} \gamma_{3}, \ldots, \tau_{\bar{m}-1}:=\mathrm{i} \gamma_{m-2} \gamma_{m-1}
$$

mutually commute and, in addition, they commute with $\gamma_{1}$ and $\gamma_{m}$. Thus, for $j, k=$ $1, \ldots, \bar{m}-1$, we have

$$
\begin{array}{ll}
\tau_{j} \tau_{k}=\tau_{k} \tau_{j} & \tau_{j}^{2}=1 \\
\tau_{j} \gamma_{1}=\gamma_{1} \tau_{j} & \tau_{j} \gamma_{m}=\gamma_{m} \tau_{j} .
\end{array}
$$

So we can choose a set of simultaneous eigenvectors of $\tau_{j}$ with eigenvalues $\rho_{j}= \pm 1$. We denote by $\vec{\rho}=\left(\rho_{1}, \ldots, \rho_{\bar{m}-1}\right)$ the collection of simultaneous eigenvalues of $\tau_{j}$ and we define the associated simultaneous eigenspaces by

$$
V_{\vec{\rho}}=\left\{v \in V: \tau_{i} v=\rho_{i} v\right\} .
$$

The vector space $V_{\vec{\rho}}$ is preserved by $\gamma_{1}, \gamma_{m}$ and $\tilde{\gamma}$. We use this fact to decompose $V_{\vec{\rho}}$ into its chiral parts,

$$
V_{\vec{\rho}}=V_{\vec{\rho}}^{+} \oplus V_{\vec{\rho}}^{-} \quad \text { where } \quad V_{\vec{\rho}}^{ \pm}=\left\{v \in V_{\vec{\rho}}: \tilde{\gamma} v= \pm v\right\} .
$$


Let $V_{\vec{\rho}}^{+}=\operatorname{span}\left\{v_{\vec{\rho}}\right\}$. Then since $\gamma_{m} \tilde{\gamma}=-\tilde{\gamma} \gamma_{m}$ we have

$$
V_{\vec{\rho}}=\operatorname{span}\left\{v_{\vec{\rho}}, \gamma_{m} v_{\vec{\rho}}\right\} .
$$

The vector spaces $V_{\vec{\rho}}$ provide the decomposition

$$
V=\bigoplus_{\vec{\rho}} V_{\vec{\rho}} \quad \operatorname{dim} V_{\vec{\rho}}=2
$$

and the problem completely decouples into two-dimensional spaces.

On $V_{\vec{\rho}}$ one easily computes, using the definition $\epsilon(\vec{\rho})=\rho_{1} \times \cdots \times \rho_{\bar{m}-1}$,

$$
\gamma_{1}=\epsilon(\vec{\rho})\left(\begin{array}{cc}
0 & \mathrm{i} \\
-\mathrm{i} & 0
\end{array}\right) \quad \gamma_{m}=\left(\begin{array}{cc}
0 & 1 \\
1 & 0
\end{array}\right) \quad \tilde{\gamma}=\left(\begin{array}{cc}
1 & 0 \\
0 & -1
\end{array}\right)
$$

thus reproducing the two-dimensional Pauli matrices up to the standard sign ambiguity.

We note that the kernel of $\Pi_{-}$is determined by the eigenvectors of $\chi$. Thus to establish (3.1) we shall need explicit representations of $\chi$ and $\tilde{q}(\xi, \mu)$ acting on $V_{\vec{\rho}}$. To calculate $\tilde{q}(\xi, \mu)$ it is possible to choose coordinates such that $\xi_{2}=\cdots=\xi_{m-1}=0$. It is then easy to see that

$$
\chi=\mathrm{i}\left(\begin{array}{cc}
0 & \mathrm{e}^{\theta} \\
-\mathrm{e}^{-\theta} & 0
\end{array}\right) \quad \tilde{q}(\xi, \mu)=\left(\begin{array}{cc}
\epsilon(\rho) \xi_{1} & \mu \\
\mu & -\epsilon(\rho) \xi_{1}
\end{array}\right) .
$$

The eigenvectors of $\chi$ follow,

$$
\chi\left(\begin{array}{c}
\varrho \\
-\mathrm{ie}^{-\theta}
\end{array}\right)=\varrho\left(\begin{array}{c}
\varrho \\
-\mathrm{ie}^{-\theta}
\end{array}\right)
$$

and we compute

$$
\tilde{q}(\xi, \mu)\left(\begin{array}{c}
\varrho \\
-\mathrm{ie}^{-\theta}
\end{array}\right)=\left(\begin{array}{c}
\epsilon(\vec{\rho}) \varrho \xi_{1}-\mathrm{i} \mu \mathrm{e}^{-\theta} \\
\mu \varrho+\mathrm{i} \epsilon(\vec{\rho}) \xi_{1} \mathrm{e}^{-\theta}
\end{array}\right)
$$

where $\varrho= \pm 1$. Assertion (1) holds if these are not multiples of each other, that is if

$$
\operatorname{det}\left(\begin{array}{cc}
\epsilon(\vec{\rho}) \varrho \xi_{1}-\mathrm{i} \mu \mathrm{e}^{-\theta} & \varrho \\
\mu \varrho+\mathrm{i} \epsilon(\vec{\rho}) \xi_{1} \mathrm{e}^{-\theta} & -\mathrm{ie}^{-\theta}
\end{array}\right)=-2 \mathrm{i} \epsilon(\vec{\rho}) \varrho \xi_{1} \mathrm{e}^{-\theta}-\mu\left(1+\mathrm{e}^{-2 \theta}\right) \neq 0 .
$$

Since the first term is purely imaginary and $\mu \notin \mathrm{iR}-\{0\}$ this proves assertion (1).

In fact, to prove what we announced (i.e. $\Pi_{-}$is an isomorphism from $V_{-}^{\tilde{q}}(\xi, \lambda)$ to $\left.\mathcal{W}=\operatorname{Range}\left(\Pi_{-}\right)\right)$, it is enough to consider only $\varrho=+1$. But since $V_{-}^{\tilde{q}}(-\xi,-\lambda)=V_{+}^{\tilde{q}}(\xi, \lambda)$, considering $\rho= \pm 1$ gives the same condition.

Although assertion (2) follows from assertion (1) and lemma 1.11.2 (b) in [21], we prefer to give a second proof showing that the boundary operator $\mathcal{B}$ involves tangential derivatives. This makes apparent that the chiral boundary conditions are non-standard boundary conditions.

\section{Ellipticity of the second-order boundary problem}

When considering spectral properties of the square of the operator of Dirac type, $P^{2}=$ $\left(\mathrm{i} \gamma_{j} \nabla_{j}\right)^{2}$, the boundary condition imposed through $\mathcal{B}$ is

$$
\begin{aligned}
& \left.\Pi_{-} \psi\right|_{\partial M}=0 \\
& \left.\Pi_{-} \gamma_{j} \nabla_{j} \psi\right|_{\partial M}=0 .
\end{aligned}
$$

The second boundary condition (4.2) can be rewritten as an oblique boundary condition involving tangential derivatives. To this end note

$$
\begin{aligned}
& \gamma_{m} \Pi_{\mp}=\Pi_{ \pm}^{\star} \gamma_{m} \\
& \gamma_{a} \Pi_{\mp}=\Pi_{\mp}^{\star} \gamma_{a}
\end{aligned}
$$


with the tangential $\gamma$-matrices $\gamma_{a}$, where $a=1, \ldots, m-1$. This allows the boundary condition (4.2) to be written as

$$
\begin{aligned}
0 & =\left.\Pi_{-}\left(-\mathrm{i} \gamma_{j} \nabla_{j}\right) \psi\right|_{\partial M}=\left.\mathrm{i} \gamma_{m} \Pi_{-}\left(-\mathrm{i} \gamma_{j} \nabla_{j}\right) \psi\right|_{\partial M} \\
& =\left.\gamma_{m} \Pi_{-}\left(\gamma_{m} \nabla_{m}+\gamma_{a} \nabla_{a}\right) \psi\right|_{\partial M}=\left.\left(\Pi_{+}^{\star} \nabla_{m}+\gamma_{m} \gamma_{a} \Pi_{-}^{\star} \nabla_{a}\right) \psi\right|_{\partial M} \\
& =\left.\left(\Pi_{+}^{\star} \nabla_{m}+\gamma_{m} \gamma_{a} \Pi_{-}^{\star} \nabla_{a}\right)\left(\Pi_{-}+\Pi_{+}\right) \psi\right|_{\partial M}
\end{aligned}
$$

where $\nabla_{m}$ is the interior normal derivative. The boundary condition contains tangential derivatives and the conditions imposed through $\mathcal{B}$ could thus be termed of mixed oblique type.

Proof of (2). To study the ellipticity of the boundary value problem, we introduce the 'partial' leading symbol of $P^{2}$

$$
\sigma_{L}\left(y, x_{m}, \omega,-\mathrm{i} \partial_{m}, \lambda\right)=-\partial_{m}^{2}+\omega^{2}-\lambda
$$

and the graded symbol, $\sigma_{g}$, of $\mathcal{B}$

$$
\sigma_{g}=\left(\begin{array}{cc}
\Pi_{-} & 0 \\
-\gamma_{a} \omega_{a} \Pi_{-}^{\star} & \mathrm{i} \gamma_{m} \Pi_{+}^{\star}
\end{array}\right) .
$$

Strong ellipticity requires that the problem

$$
\sigma_{L}\left(y, x_{m}, \omega,-\mathrm{i} \partial_{m}, \lambda\right) \Psi\left(y, x_{m}, \omega, \lambda\right)=0
$$

with

$$
\Psi \rightarrow_{r \rightarrow \infty} 0
$$

and

$$
\left.\left.\sigma_{g}\left(\begin{array}{c}
\Psi \\
\partial_{m} \Psi
\end{array}\right)\right\rfloor_{r=0}=\left(\begin{array}{c}
\Pi_{-} \alpha \\
\mathrm{i} \gamma_{m} \Pi_{+}^{\star} \partial_{m} \alpha
\end{array}\right)\right\rfloor_{r=0}
$$

has a unique solution.

Now, the solutions to (4.4) and (4.5) are

$$
\Psi\left(x_{m}, \omega, \lambda\right)=\Psi_{0} \exp \left(-\Lambda x_{m}\right)
$$

where $\Lambda=+\sqrt{\omega^{2}-\lambda}$. Note that $\operatorname{Re}(\Lambda)>0$ for $\lambda \in \mathbf{C}-\mathbf{R}_{+}$.

The condition (4.6), when applied to them, reads

$$
\left(\begin{array}{cc}
\Pi_{-} & 0 \\
-\gamma_{a} \omega_{a} \Pi_{-}^{\star} & \mathrm{i} \gamma_{m} \Pi_{+}^{\star}
\end{array}\right)\left(\begin{array}{c}
\Psi_{0} \\
-\Lambda \Psi_{0}
\end{array}\right)=\left(\begin{array}{c}
\Pi_{-} \alpha \\
-\mathrm{i} \gamma_{m} \Pi_{+}^{\star} \Lambda \alpha
\end{array}\right) .
$$

This gives a system of two equations. After multiplying the second one by $\mathrm{i} \gamma_{m}$, and using $\Psi_{0}=\Pi_{-} \Psi_{0}+\Pi_{+} \Psi_{0}$, one obtains

$$
\Pi_{-} \Psi_{0}=\Pi_{-} \alpha
$$

and

$\left(-\mathrm{i} \gamma_{m}\left(\gamma_{a} \omega_{a}\right) \Pi_{-}^{\star} \Pi_{+}+\Lambda \Pi_{+}^{\star} \Pi_{+}\right) \Psi_{0}=\Lambda \Pi_{+}^{\star} \alpha-\left(-\mathrm{i} \gamma_{m}\left(\gamma_{a} \omega_{a}\right) \Pi_{-}^{\star} \Pi_{-}+\Lambda \Pi_{+}^{\star} \Pi_{-}\right) \Psi_{0}$.

We use (2.1) and substitute (4.7) into (4.8) to see

$$
\begin{aligned}
& \exp (-\theta \tilde{\gamma})\left[-\mathrm{i} \gamma_{m}\left(\gamma_{a} \omega_{a}\right) \sinh (\theta \tilde{\gamma})+\Lambda \cosh (\theta \tilde{\gamma})\right] \Pi_{+} \Psi_{0} \\
& =\Lambda \Pi_{+}^{\star} \alpha-\left[-\mathrm{i} \gamma_{m}\left(\gamma_{a} \omega_{a}\right) \cosh (\theta \tilde{\gamma})+\Lambda \sinh (\theta \tilde{\gamma})\right] \exp (-\theta \tilde{\gamma}) \Pi_{-} \alpha .
\end{aligned}
$$

So, the problem has a unique solution if the matrix

$$
M=-\mathrm{i} \gamma_{m}\left(\gamma_{a} \omega_{a}\right) \sinh (\theta \tilde{\gamma})+\Lambda \cosh (\theta \tilde{\gamma})=A \sinh (\theta)+\Lambda \cosh (\theta \tilde{\gamma})
$$

is non-singular, where we introduced $A=-\mathrm{i} \tilde{\gamma} \gamma_{m} \gamma_{a} \omega_{a}$. 
But $A^{\star}=A$; so, it is diagonalizable. Moreover, $A^{2}=\left(\sum_{a} \omega_{a}^{2}\right)$ 1. As a consequence, $A$ has eigenvalues $\pm \sqrt{\sum_{a} \omega_{a}^{2}}$, except in two dimensions, where $A$ is proportional to the identity. Then, the determinant of $M$ can be evaluated in the basis of eigenvectors of $A$, and in all cases, $\operatorname{det} M$ can be seen to vanish if $\lambda=\frac{\sum_{a} \omega_{a}^{2}}{\cosh ^{2} \theta}$. For $\lambda=0$, the determinant can only vanish if $\omega_{a}=0$. Otherwise, it can only happen for $\lambda \in \mathbf{R}_{+}$, which proves strong ellipticity in $\mathbf{C}-\mathbf{R}_{+}$, and any even dimension. This completes the proof of theorem 2.1.

\section{Heat kernel in an infinite cylinder}

In what follows, we present the heat kernel for $\left(P^{2}, \mathcal{B}\right)$ in an infinite cylinder $\mathcal{M}=\mathbf{R}_{+} \times \mathcal{N}$ of any even dimension. By cylinder we mean that the metric is of the type $\mathrm{d} s^{2}=\mathrm{d} x_{m}^{2}+\mathrm{d} s_{\mathcal{N}}^{2}$, where $\mathrm{d} s_{\mathcal{N}}^{2}$ is the metric of the closed boundary $\mathcal{N}$.

In order to determine the heat kernel, it is useful to note that the chiral bag boundary conditions in equations (4.1) and (4.2) are equivalent, for each eigenvalue of the tangential part $B$ of the operator $P$, to Dirichlet boundary conditions on part of the fibre, and Robin (modified Neumann) on the rest.

In fact, let us first note that the operators $\mathcal{P}_{+}=\frac{\Pi_{+} \Pi_{+}^{\star}}{\cosh ^{2} \theta}$ and $\mathcal{P}_{-}=\frac{\Pi_{-}^{\star} \Pi_{-}}{\cosh ^{2} \theta}$ are self-adjoint projectors, and they satisfy $\mathcal{P}_{+}+\mathcal{P}_{-}=1$ splitting $V$ into two complementary subspaces.

Let $\xi=x_{m}-x_{m}^{\prime}$ and $\eta=x_{m}+x_{m}^{\prime}$ and as before, let $y=\left(y_{1}, y_{2}, \ldots, y_{m-1}\right)$ be the coordinates on the boundary and $x=\left(y, x_{m}\right)$. If we call $\phi_{\omega}(y)$ the eigenspinors of the operator $B=\tilde{\gamma} \gamma_{m} \gamma_{a} \partial_{a}$ (with $a=1,2, \ldots, m-1$ ) corresponding to the eigenvalue $\omega$, normalized such that

$$
\sum_{\omega} \phi_{\omega}^{\star}(y) \phi_{\omega}\left(y^{\prime}\right)=\delta^{m-1}\left(y-y^{\prime}\right)
$$

with $\delta^{m-1}$ the Dirac delta function, and

$$
\int_{\partial M} \mathrm{~d} y \phi_{\omega}^{\star}(y) \phi_{\omega}(y)=1
$$

we can expand $\psi\left(y, x_{m}\right)=\sum_{\omega} f_{\omega}\left(x_{m}\right) \phi_{\omega}(y)$. If $\psi=\mathcal{P}_{+} \psi$, then the condition (4.1) is identically satisfied, and only (4.3) must be imposed at the boundary which, for each $\omega$, reduces to

$$
\cosh \theta \mathrm{e}^{-\theta \tilde{\gamma}}\left(\partial_{m}+\omega \tanh \theta\right) f_{\omega}=0 .
$$

Since the factor to the left of the parenthesis is invertible, this is nothing but a Robin boundary condition.

In the subspace $\psi=\mathcal{P}_{-} \psi$, the boundary condition (4.1) reduces to

$$
\cosh \theta \mathrm{e}^{\theta \tilde{\gamma}} f_{\omega}=0
$$

while (4.3) requires

$$
\omega f_{\omega}=0 .
$$

Thus, in this subspace, both boundary conditions are nothing but homogeneous Dirichlet ones.

As a consequence, the complete heat kernel can be written as a Dirichlet heat kernel on $\mathcal{P}_{-} V$ and a Robin heat kernel on $\mathcal{P}_{+} V$. For the convenience of the reader we make the single ingredients explicit [22] and write

$$
\begin{aligned}
K\left(t ; x, x^{\prime}\right) & =K\left(t ; x, x^{\prime}\right)\left(\mathcal{P}_{-}+\mathcal{P}_{+}\right) \\
& =\frac{1}{\sqrt{4 \pi t}} \sum_{\omega} \phi_{\omega}^{\star}\left(y^{\prime}\right) \phi_{\omega}(y) \mathrm{e}^{-\omega^{2} t}\left(\mathrm{e}^{\frac{-\xi^{2}}{4 t}}-\mathrm{e}^{\frac{-\eta^{2}}{4 t}}\right) \mathcal{P}_{-}
\end{aligned}
$$




$$
\begin{aligned}
& +\frac{1}{\sqrt{4 \pi t}} \sum_{\omega} \phi_{\omega}^{\star}\left(y^{\prime}\right) \phi_{\omega}(y) \mathrm{e}^{-\omega^{2} t}\left\{\left(\mathrm{e}^{\frac{-\xi^{2}}{4 t}}+\mathrm{e}^{\frac{-\eta^{2}}{4 t}}\right)\right. \\
& \left.+2 \sqrt{\pi t} \omega \tanh \theta \mathrm{e}^{\omega^{2} t \tanh ^{2} \theta-\omega \eta \tanh \theta} \operatorname{erf} c\left[u_{\omega}(\eta, t)\right]\right\} \mathcal{P}_{+} \\
& =\frac{1}{\sqrt{4 \pi t}} \sum_{\omega} \phi_{\omega}^{\star}\left(y^{\prime}\right) \phi_{\omega}(y) \mathrm{e}^{-\omega^{2} t}\left\{\left(\mathrm{e}^{\frac{-\xi^{2}}{4 t}}-\mathrm{e}^{\frac{-\eta^{2}}{4 t}}\right) \mathbf{1}\right. \\
& \left.+\frac{2 \Pi_{+} \Pi_{+}^{\star}}{\cosh ^{2}(\theta)}\left[1+\sqrt{(\pi t)} \omega \tanh \theta \mathrm{e}^{u_{\omega}(\eta, t)^{2}} \operatorname{erf} c\left[u_{\omega}(\eta, t)\right]\right] \mathrm{e}^{\frac{-\eta^{2}}{4 t}}\right\}
\end{aligned}
$$

where $u_{\omega}(\eta, t)=\frac{\eta}{\sqrt{4 t}}-\sqrt{t} \omega \tanh (\theta)$, and

$$
\operatorname{erf} c(x)=\frac{2}{\sqrt{\pi}} \int_{x}^{\infty} \mathrm{d} \xi \mathrm{e}^{-\xi^{2}}
$$

is the complementary error function. Note that (5.1) is a direct generalization of the heat kernel given in [6] for the two-dimensional case, which, in turn, coincides with the Fourier transform of equation (101) in [4] for an anti-periodic boundary fibre.

\section{Meromorphic properties of the zeta function}

Let us now analyse the boundary contributions to the global zeta function related to (5.1). We first note that global quantities are necessarily divergent due to the non-compact nature of our manifold $\mathcal{M}=\mathbf{R}_{+} \times \mathcal{N}$. This is not a severe problem because the result (5.1) allows us to easily identify the bulk term leading to a divergent contribution when integrated. In particular, it is the first term in (5.1) that represents the heat kernel on the manifold $\mathbf{R} \times \mathcal{N}$. In the following, without changing the notation, we will first ignore this term and this will allow us to determine the boundary contributions to the global zeta function. Alternatively, as we will show afterwards, one can introduce a localizing function of compact support in (5.1) such that the trace gives a finite result.

Let us consider the trace of (5.1) ignoring the first term. It is convenient to perform the Dirac trace (tr) first. Since

$$
\operatorname{tr}\left(\frac{2 \Pi_{+} \Pi_{+}^{\star}}{\cosh ^{2}(\theta)}\right)=2^{\bar{m}}
$$

the trace of the 'boundary' heat kernel reduces to

$\operatorname{Tr} K(t ; x, x)=\frac{2^{\bar{m}}}{2} \sum_{\omega} \omega \tanh \theta \mathrm{e}^{-\omega^{2} t} \int_{0}^{\infty} \mathrm{d} x_{m} \operatorname{erf} c\left[u_{\omega}\left(2 x_{m}, t\right)\right] \exp \left(\frac{-x_{m}^{2}}{t}+u_{\omega}^{2}\left(2 x_{m}, t\right)\right)$

where the second and third term in (5.1) have cancelled each other. Now, using that

$$
\begin{aligned}
& -\frac{1}{2} \frac{\partial}{\partial x_{m}}\left[\mathrm{e}^{-x_{m}^{2} / t+u_{\omega}^{2}\left(2 x_{m}, t\right)} \operatorname{erf} c\left[u_{\omega}\left(2 x_{m}, t\right)\right]\right] \\
& =\mathrm{e}^{-x_{m}^{2} / t}\left[\frac{1}{\sqrt{\pi t}}+\omega \tanh \theta \mathrm{e}^{u_{\omega}^{2}\left(2 x_{m}, t\right)} \operatorname{erf} c\left[u_{\omega}\left(2 x_{m}, t\right)\right]\right]
\end{aligned}
$$

we get

$$
\begin{aligned}
\operatorname{Tr} K(t ; x, x) & =\frac{2^{\bar{m}}}{4} \sum_{\omega} \mathrm{e}^{-\omega^{2} t}\left[\mathrm{e}^{u_{\omega}^{2}(0, t)} \operatorname{erf} c\left[u_{\omega}(0, t)\right]-1\right] \\
& =\frac{2^{\bar{m}}}{4} \sum_{\omega}\left[\mathrm{e}^{\frac{-\omega^{2} t}{\cosh ^{2} \theta}}[1+\operatorname{erf}(\omega \sqrt{t} \tanh \theta)]-\mathrm{e}^{-\omega^{2} t}\right] .
\end{aligned}
$$

Here we used erf $(x)=-\operatorname{erf}(-x)=1-\operatorname{erf} c(x)$. 
Now, we can Mellin transform this trace, to obtain the 'boundary' zeta function of the square of the Dirac operator in the infinite cylinder

$$
\begin{aligned}
\zeta\left(s, P^{2}\right)=\frac{2^{\bar{m}}}{4 \Gamma(s)} \sum_{\omega} \int_{0}^{\infty} \mathrm{d} t t^{s-1}\left[\mathrm{e}^{\frac{-\omega^{2} t}{\cosh ^{2} \theta}}-\mathrm{e}^{-\omega^{2} t}\right] \\
\quad+\frac{2^{\bar{m}}}{4 \Gamma(s)} \sum_{\omega} \int_{0}^{\infty} \mathrm{d} t t^{s-1} \mathrm{e}^{\frac{-\omega^{2} t}{\cosh ^{2} \theta}} \operatorname{erf}(\omega \sqrt{t} \tanh \theta)=\zeta_{1}\left(s, P^{2}\right)+\zeta_{2}\left(s, P^{2}\right) .
\end{aligned}
$$

The first contribution can be readily seen to be

$$
\zeta_{1}\left(s, P^{2}\right)=\frac{1}{4}\left(\cosh ^{2 s} \theta-1\right) \zeta\left(s, B^{2}\right)
$$

where $B$ is the operator defined in section 5 .

As for the second contribution to (6.1), it is given by

$$
\zeta_{2}\left(s, P^{2}\right)=\frac{2^{\bar{m}}}{4 \Gamma(s)} \sum_{\omega} \int_{0}^{\infty} \mathrm{d} t t^{s-1} \mathrm{e}^{\frac{-\omega^{2} t}{\cosh ^{2} \theta}} \frac{2}{\sqrt{\pi}} \int_{0}^{(\omega \sqrt{t} \tanh \theta)} \mathrm{d} \xi \mathrm{e}^{-\xi^{2}} .
$$

After changing variables according to $y=\frac{\xi \cosh \theta}{\sqrt{t} \omega}$, and interchanging integrals, one finally gets

$$
\begin{aligned}
\zeta_{2}\left(s, P^{2}\right) & =\frac{2^{\bar{m}} \Gamma\left(s+\frac{1}{2}\right)}{4 \Gamma(s)} \cosh ^{2 s} \theta \sum_{\omega} \operatorname{sign}(\omega)\left(\omega^{2}\right)^{-s} \frac{2}{\sqrt{\pi}} \int_{0}^{\sinh \theta} \mathrm{d} y\left(1+y^{2}\right)^{-s-\frac{1}{2}} \\
& =\frac{\Gamma\left(s+\frac{1}{2}\right)}{4 \Gamma(s)} \cosh ^{2 s} \theta \eta(2 s, B) \frac{2}{\sqrt{\pi}} \int_{0}^{\sinh \theta} \mathrm{d} y\left(1+y^{2}\right)^{-s-\frac{1}{2}} \\
& =\frac{1}{2 \sqrt{\pi}} \frac{\Gamma\left(s+\frac{1}{2}\right)}{\Gamma(s)} \sinh \theta \cosh ^{2 s} \theta \eta(2 s, B)_{2} F_{1}\left(\frac{1}{2}, \frac{1}{2}+s, \frac{3}{2} ;-\sinh ^{2} \theta\right) .
\end{aligned}
$$

The structure of the zeta function is similar to the structure found for spectral boundary conditions, see e.g., [10]. In particular, the analysis of the zeta function on $\mathcal{M}$ has been reduced to the analysis of the zeta and eta function on $\mathcal{N}$.

As already commented, from (6.2) and (6.3) one can determine the positions of the poles and corresponding residues for the zeta function in any cylindrical product manifold, in terms of the meromorphic structure of the zeta and eta functions of the boundary operator. For the rightmost poles, explicit results can be given in terms of the geometry of the boundary $\mathcal{N}$. For example, for $s=(m-1) / 2$ we see that

$$
\begin{aligned}
\operatorname{Res} \zeta_{1}\left(\frac{m-1}{2}, P^{2}\right) & =\frac{1}{4}\left(\cosh ^{m-1} \theta-1\right) \operatorname{Res} \zeta\left(\frac{m-1}{2}, B^{2}\right) \\
& =\frac{1}{4}\left(\cosh ^{m-1} \theta-1\right) \frac{(4 \pi)^{-(m-1) / 2}}{\Gamma\left(\frac{m-1}{2}\right)} 2^{\bar{m}} \operatorname{Vol}(\mathcal{N}) .
\end{aligned}
$$

Because $\zeta_{2}$ does not contribute, given $\eta(2 s, B)$ is regular at $s=(m-1) / 2$ [21], this equals $\operatorname{Res} \zeta(s, P)$ and is the result expected from the calculation on the ball [23]. For $\theta=0$ the residue disappears as is known to happen for the standard local bag boundary conditions [21]. Further results can be obtained by using theorem 4.4.1 of [21]. Given we considered the case without 'potential', it is immediate that

$$
\operatorname{Res} \zeta\left(\frac{m-2}{2}, P^{2}\right)=0 \text {. }
$$

Also, for the particular case of $s=0$, the fact that $\zeta\left(s, B^{2}\right)$ and $\eta(2 s, B)$ are regular at $s=0$ shows that $\zeta\left(0, P^{2}\right)=\zeta(0, P)=0$. 
Given the local heat kernel (5.1), a local version of the results of this section is easily obtained. To this end, we use a localizing function with compact support near the boundary, such that its normal derivatives at the boundary vanish,

$$
\left.\frac{\partial^{n}}{\partial x_{m}^{n}} f\left(y, x_{m}\right)\right|_{x_{m}=0}=0 \quad n \in \mathbb{N} \text {. }
$$

Furthermore, we let $\tilde{P}^{2}$ denote the operator $P^{2}$ on the double $\mathbf{R} \times \mathcal{N}$ of $\mathbf{R}_{+} \times \mathcal{N}$, and we extend the localizing function as an even function to the double. We use the notation $f=f\left(y, x_{m}\right), f_{\mathcal{N}}=f\left(y, x_{m}=0\right)$ and $\tilde{f}$ for $f$ on the double. Introducing the local versions $\zeta\left(\tilde{f}, s, \tilde{P}^{2}\right), \zeta\left(f, s, P^{2}\right), \zeta\left(f_{\mathcal{N}}, s, B^{2}\right)$ and $\eta\left(f_{\mathcal{N}}, 2 s, B\right)$ of the zeta functions and the eta function, (5.1) and previous calculations show that the following theorem holds:

\section{Theorem 6.1.}

$$
\begin{aligned}
\Gamma(s) \zeta\left(f, s, P^{2}\right) & =\Gamma(s)\left\{\frac{1}{2} \zeta\left(\tilde{f}, s, \tilde{P}^{2}\right)+\frac{1}{4}\left(\cosh ^{2 s} \theta-1\right) \zeta\left(f_{\mathcal{N}}, s, B^{2}\right)\right. \\
& +\frac{1}{2 \sqrt{\pi}} \frac{\Gamma(s+1 / 2)}{\Gamma(s)} \sinh \theta \cosh ^{2 s} \theta \\
& \left.\times{ }_{2} F_{1}\left(\frac{1}{2}, \frac{1}{2}+s, \frac{3}{2},-\sinh ^{2} \theta\right) \eta\left(f_{\mathcal{N}}, 2 s, B\right)\right\}+h(s)
\end{aligned}
$$

where $h(s)$ is an entire function.

This result parallels theorem 2.1 in [10] for spectral boundary conditions.

\section{Conclusions}

In this paper we have considered the influence of generalized bag boundary conditions on the heat kernel and the zeta function. In order to guarantee certain structural properties we have first shown the strong ellipticity of the boundary conditions. Work by Seeley $[24,25]$ then shows that the standard heat kernel expansion holds and so the zeta function can have only simple poles at $s=m / 2,(m-1) / 2, \ldots, 1 / 2$, and $s=-(2 l+1) / 2, l \in \mathbb{N}$. This is the main result of this paper.

Based on the strong ellipticity one might envisage the determination of the leading heat kernel coefficients for generalized bag boundary conditions as they are needed for the calculation of effective actions in gauge theories in Euclidean bags [3]. Special case calculations can serve to restrict the general form that coefficients can have, cylindrical manifolds providing a valuable example. Here, for $P=\mathrm{i} \gamma_{j} \nabla_{j}$, we have expressed the heat kernel and the zeta function of the associated second-order operator on $\mathcal{M}=\mathbf{R}_{+} \times \mathcal{N}$ in terms of the boundary data on $\mathcal{N}$. In fact, this result, under certain restrictions, can be straightforwardly generalized to $P=\mathrm{i} \gamma_{j} \nabla_{j}-\phi$. In order that a separation of variables as presented succeeds we need $\partial_{x_{m}} \phi=0$ and $\left\{\gamma_{m}, \phi\right\}=\{\tilde{\gamma}, \phi\}=0$. If this is satisfied, equations (6.2) and (6.3) remain valid, once the operator $B$ incorporates the potential, $B=\tilde{\gamma} \gamma_{m}\left(\gamma_{a} \nabla_{a}-\mathrm{i} \phi\right)$. We have thus a particular case involving a potential and Riemannian curvature and various restrictions on heat kernel coefficients will follow.

\section{Acknowledgments}

Research of CGB and EMS was partially supported by CONICET(PIP 0459/98) and UNLP(11/X298). Research of PG was partially supported by the MPI (Leipzig, Germany). 
KK acknowledges support by the Baylor University Summer Sabbatical Program and by the MPI (Leipzig, Germany).

\section{Appendix. $\gamma$-matrices}

Let $m=2 \bar{m}$ be the dimension of a Riemannian manifold. We denote by $\gamma_{j}^{(m)}, j=1, \ldots, m$, the self-adjoint $\gamma$-matrices projected along some $m$-bein system. These are defined inductively by

$$
\begin{array}{ll}
\gamma_{j}^{(m)}=\left(\begin{array}{cc}
0 & \mathrm{i} \gamma_{j}^{(m-2)} \\
-\mathrm{i} \gamma_{j}^{(m-2)} & 0
\end{array}\right) \quad j=1, \ldots, m-1 \\
\gamma_{m}^{(m)}=\left(\begin{array}{ll}
0 & 1 \\
1 & 0
\end{array}\right) & \gamma_{m+1}^{(m)}=\left(\begin{array}{cc}
1 & 0 \\
0 & -1
\end{array}\right)
\end{array}
$$

starting from the Pauli matrices

$$
\gamma_{1}^{(2)}=\left(\begin{array}{cc}
0 & \mathrm{i} \\
-\mathrm{i} & 0
\end{array}\right) \quad \gamma_{2}^{(2)}=\left(\begin{array}{ll}
0 & 1 \\
1 & 0
\end{array}\right) \quad \gamma_{3}^{(2)}=\left(\begin{array}{cc}
1 & 0 \\
0 & -1
\end{array}\right) .
$$

The $\gamma$-matrices satisfy the Clifford anti-commutation formula

$$
\gamma_{j}^{(m)} \gamma_{k}^{(m)}+\gamma_{k}^{(m)} \gamma_{l}^{(m)}=2 \delta_{k l}
$$

In the main body of the paper we will simplify the notation and we will not indicate the dimension explicitly. In addition, we set

$$
\gamma_{m+1}^{(m)}=\tilde{\gamma}=(-\mathrm{i})^{\bar{m}} \gamma_{1} \ldots \gamma_{m}
$$

which is the generalization of ' $\gamma_{5}$ ' to arbitrary even dimension.

\section{References}

[1] Kirsten K 2001 Spectral Functions in Mathematics and Physics (Boca Raton, FL: Chapman \& Hall/CRC)

[2] Hrasko P and Balog J 1984 The fermion boundary condition and the theta angle in QED in two-dimensions Nucl. Phys. B 245 118-26

[3] Wipf A and Dürr S 1995 Gauge theories in a bag Nucl. Phys. B 443 201-32

[4] Dürr S and Wipf A 1997 Finite temperature Schwinger model with chirality breaking boundary conditions Ann. Phys., NY $255333-61$

[5] Dürr S 1999 Aspects of quasi-phasestructure of the Schwinger model on a cylinder with broken chiral symmetry Ann. Phys., NY 273 1-36

[6] Beneventano C G, Santangelo E M and Wipf A 2002 Spectral asymmetry for bag boundary conditions J. Phys. A: Math. Gen. 35 9343-54

[7] Esposito G and Kirsten K 2002 Chiral bag boundary conditions on the ball Phys. Rev. D 66085014

[8] Dowker J S and Kirsten K 1999 The a (3/2) heat kernel coefficient for oblique boundary conditions Class. Quantum Grav. 16 1917-36

[9] Avramidi I G and Esposito G 1999 Gauge theories on manifolds with boundary Commun. Math. Phys. 200 495-543

[10] Grubb G and Seeley R T 1996 Zeta and eta functions for Atiyah-Patodi-Singer operators J. Geom. Anal. 6 31-77

[11] Elizalde E and Vassilevich D V 1999 Heat kernel coefficients for Chern-Simons boundary conditions in QED Class. Quantum Grav. 16 813-23

[12] Elizalde E, Lygren M and Vassilevich D V 1996 Antisymmetric tensor fields on spheres: functional determinants and nonlocal counterterms J. Math. Phys. 37 3105-17

[13] Elizalde E, Lygren M and Vassilevich D V 1997 Zeta function for the Laplace operator acting on forms in a ball with gauge boundary conditions Commun. Math. Phys. 183 645-60

[14] Śniatycki J and Schwarz G 1994 The existence and uniqueness of solutions of Yang-Mills equations with bag boundary conditions Commun. Math. Phys. 159 593-604 
[15] McKellar B H J, Stephenson G J, Jr and Thomson M J 1993 The Dirac equation in Kerr spacetime, spheroidal coordinates and the MIT bag model of hadrons J. Phys. A: Math. Gen. 26 3649-57

[16] Freedman B and Krapchev V 1976 Effects of boundary conditions on massless two-dimensional electrodynamics in a static bag Phys. Rev. D 14 566-77

[17] Geffen D A and Suura H 1977 Solutions to a gauge-invariant, equal-time two-body wave equation. Light-mass quark-antiquark system Phys. Rev. D 16 3305-19

[18] Colanero K and Chu M-C 2002 Analytical solution of the dynamical spherical MIT bag J. Phys. A: Math. Gen. 35 993-9

[19] Ansoldi S, Castro C and Spallucci E 2001 Chern-Simons hadronic bag from quenched large-N QCD Phys. Lett. B 504 174-80

[20] Kravchenko V V 1995 On a biquaternionic bag model Z. Anal. Anwend. 14 3-14

[21] Gilkey P B 1995 Invariance Theory, the Heat Equation and the Atiyah-Singer Index Theorem (Boca Raton, FL: CRC Press)

[22] Carslaw H S and Jaeger J C 1986 Conduction of Heat in Solids (Oxford: Clarendon)

[23] Kirsten K 2002 Heat kernel asymptotics: more special case calculations Nucl. Phys. B (Proc. Suppl.) 104 119-26

[24] Seeley R T 1968 Complex powers of an elliptic operator Singular Integrals (Chicago 1966) (Proc. Sympos. Pure Math. vol 10) (Providence, RI: American Mathematics Society) pp 288-307

[25] Seeley R T 1969 Analytic extension of the trace associated with elliptic boundary problems Am. J. Math. 91 963-83 